\title{
EP-34
}

\section{Recurrent liver metastasis in ovarian cancer}

\author{
Harshita DUBEY, Amar RANJAN*
}

Department of Laboratory Oncology, DR. BRA Institute-Rotary Cancer Hospital, All India Institute of Medical Sciences, New Delhi, India

Introduction: The most common sites of metastasis in ovarian cancer is liver, peritoneum, and lymph nodes resulting from hematogenous spread or secondary to liver parenchymal invasion from perihepatic peritoneal metastases.

Methods: The case was retrieved from routine reporting at our hospital.

Results: A 34-year-old female presented with lump in left side of abdomen for six months. Ultrasonography showed Left complex tubo-ovarian mass. Serum level of CA125 was $19.8 \mathrm{U} / \mathrm{mL}$. Upfront surgery was done. Histopathology showed malignant lesion, positive for WT1, P53 \& CK7 and negative for CK 20. Retroperitoneal lymph nodes and omentum were involved. The cases was diagnosed as epithelial ovarian cancer, high grade papillary serous cyst adenocarcinoma stage III. Three months after surgery repeat contrast enhanced computed tomography showed lung and liver metastasis lymphadenopathy. Patient was given 6 cycles of paclitaxel + carboplatin to achieve complete clinical response (CR). Lesions of lung, liver \& lymph node subsided. She spent 33 months of treatment free interval, after which lymph node enlargement was noticed and its fine needle aspiration cytology showed metastasis. Patient was put on to oral metronomic therapy with etoposide, cyclophosphamide, and celecoxib (COX-2 inhibitor) for 8 months, which showed CR. Again after 4 months, lesion in liver reappeared with raised CA 125, suggesting second relapse. The patient is on oral metronomic therapy till date with improved clinical condition.

Conclusions: This study highlights the need of early diagnosis, adjuvant chemotherapy with frequent follow up in this case. Liver is an early site for metastasis. Second relapse in also not uncommon. 